\title{
Common Representation of Information Flows for Dynamic Coalitions
}

\author{
I. Mozolevsky and J.S. Fitzgerald \\ School of Computing Science \\ Newcastle University \\ UK \\ \{igor.mozolevsky, john.fitzgerald\}@ncl.ac.uk
}

\begin{abstract}
We propose a formal foundation for reasoning about access control policies within a Dynamic Coalition, defining an abstraction over existing access control models and providing mechanisms for translation of those models into information-flow domain. The abstracted information-flow domain model, called a Common Representation, can then be used for defining a way to control the evolution of Dynamic Coalitions with respect to information flow.
\end{abstract}

\section{Introduction}

A Dynamic Coalition (DC) is a group of independent organisations collaborating towards the achievement of a goal that the member organisations could not achieve alone. The dynamic character comes from the fact that membership, and the capabilities of members, may change over time. The heterogeneity of the DC membership, bringing together organisations with very different policies governing access to their services, gives rise to security concerns that would normally not be associated with a well-defined static organisation. In order to collaborate effectively, resources need to be shared between the participants. Many DCs emerge through ad-hoc assembly but it is nevertheless desirable to engineer infrastructure and policies to enable aspects of the behaviour of coalitions to be verified in advance of operation.

We treat a dynamic coalition as a group of autonomous communicating agents (the coalition members) [4, 5]. Each member provides services that may be requested by other members and which may entail the granting of access to resources. Each organisation that participates in a DC may be represented as a whole as one member of the DC or as number of members. The level of abstraction in this case depends on the desired level of atomicity of an agent. For example, in the naval context an agent may represent the whole of a vessel or merely the vessel's captain.

Much of the challenge in designing information flow policies and infrastructure to support a DC lies in coping with the changing membership with the need to potentially renegotiate access and reallocate tasks from the workflow [5].

Ideally, the dynamic properties of membership should not disrupt the operation of the coalition where both availability and confidentiality are equally significant. These important requirements are often overlooked and simplified (at least for dynamically reconfigurable systems) by, for example, assuming that the operations can be suspended while the reconfiguration takes place [1, 13, 18]; in reality one cannot suspend operations while adding new agents and assessing the impact of new agents. Furthermore, as with most complex systems, security is often added after the design stage as a bolt-on, which is unacceptable where confidentiality and dependability of information is a significant factor. The problem of maintaining security properties of a dynamic coalition are further compounded by heterogeneity - having

Jeremy W. Bryans and John S. Fitzgerald (Eds.): Formal Aspects of Virtual Organisations 2009 (FAVO2009)

EPTCS 16, 2010, pp. 15-25 doi 10.4204/EPTCS.16.2 (c) I. Mozolevsky \& J. S. Fitzgerald

This work is licensed under the Creative Commons Attribution License. 
numerous agents that potentially have different and conflicting access control policies. This gives rise to the need to be able to reason about and assess the impact of dynamic changes on coalition membership.

This paper focuses on security considerations in DC and proposes the use of a Common Representation (CR) that allows coalition designers and architects to evaluate the effects of changes in membership of a coalition and interaction between agents. The common representation describes information flows between agents and resources within a DC. Functions over the CR describe ways of analysing the composition of numerous access control policies, the impact of agents joining and departing a coalition, coalition merging, and conflicts that may arise during a coalition's life.

By introducing the common representation, the paper addresses the problem of managing access controls in dynamic coalitions where there already exist access restrictions between subsets of agents without having to design yet another access control model. Having a set of algebraic expressions also allows coalition engineers to reason about the liveliness (ability of the coalition to complete its goals) and the confidentiality properties of different configurations of access control restrictions.

The CR, in turn, is a foundation block of a Security Meta-Policy (SMP) which is used to guide and control the evolution of a DC by specifying security properties that must be preserved every time there is a change in the coalition and by describing how security-related changes must be implemented.

The paper consists of three parts. First, we first consider various access control methods in use today (Section 2). Second, then we look at how the common representation is expressed using directed graphs (Section 3) and show how a selection of current access control methods can be expressed in terms of this common representation (Section 4). Finally we examine the use of the common representation to define approaches to coalition composition (Sections 5 and 6). We end with conclusions and a discussion of future work.

\section{Access Control in Coalitions}

As the information within a coalition is shared between various agents, some of whom may be competitors, the ability to guard the information against misuse and to prevent this information from leaking uncontrollably from the coalition is of paramount importance.

For the purposes of the present study we treat each DC member as a single entity. In practice, members may themselves be composed of individuals that receive data and issue responses on behalf of the member as a whole. We also assume that each individual tasks in the workflow being executed by the DC may entail invocations a single service by a single agent. We therefore assume that the workflow has been broken down to a relatively low level of granularity. We revisit these assumptions in Section 7

The most elementary form of access control is Discretionary Access Controls (DACs). In a DAC model, either the owner or the custodian, of information or resource has full discretion over who and at what level (e. g. write, read) is permitted access to the resource. While this form of access control may have its place in an organisation, the risk of information misuse and leakage is too great for DACs to be practically used in a DC.

There already exist numerous non-discretionary access control mechanisms that are used in organisations today. The non-discretionary access controls are typically predefined and are managed centrally. The two that are most prominently used are Lattice-Based Access Controls (LBACs) [17] and Role-Based Access Controls (RBACs) [16]. LBACs are very popular in military and other government organisations as this model is compatible with the 'need-to-know' security principle whereby every participant and piece of information is classified based on set criteria. While such an approach may be perfect for one organisation, different organisations have different classification criteria; for example, the US and the 
UK military have different clearance levels. If LBACs were to be used in a DC, then all participants and information would have to either be reclassified based on new common criteria or each participating organisation would have to communicate through a trusted agent that would be responsible for ensuring that only permitted information can leave the organisation. Both of these solutions are unacceptable, as the first one creates a huge overhead for each new organisation wishing to join the coalition, and the second one creates choke points which limits communication bandwidth between the agents. Additionally, non-government organisations may be unfamiliar with or not structured around LBACs, which introduces another complication for interactions between government agencies and private companies as the two are considered to have different priorities with respect to the information security - Clark and Wilson argued [7] that military is concerned with confidentiality, while for private companies integrity is more important.

RBAC is a more flexible scheme than LBAC whereby access is granted based on the role of the participant. When the agents were to interact across organisation boundaries, new roles would have to be created in each organisation based on the involvement of the interaction. RBACs still need to be controlled through a centralised authorisation system, which leads to unreasonably high administration overhead for large systems, especially when the participants may not necessarily be well-known to the administering authority. Furthermore, without frequent reviews, in the real world, RBACs are known to have a privilege accumulation problem, where participants retain privileges that are no longer required and may lead to information leakage when misused.

Freudenthal et al. [10] have attempted to address some of the drawbacks of the RBACs in distributed environments through Distributed Role-Based Access Controls (dRBAC) as part of a larger project on Distributed Coalition Infrastructure [11]. While dRBAC is a significant step in terms of ensuring a degree of trust and distributing the responsibility for creation of roles, this approach relies on creating new roles for participants and is still prone to privilege accumulation.

In the context of DCs, all of the above methods of access control require conversion of agents' policies from one to another. Performing such conversion will require a set amount of time during which the coalition membership must remain constant; depending on the rate of change of membership, such constraint may be unfeasible. On top of that, none of the aforementioned access control methods address security policy compatibility across the participants of dynamic coalitions, and providing strong guarantees that information will not traverse through organisational boundaries and end up in the wrong hands is nearly impossible without such analysis.

There already exist security policy description languages like Ponder [9], EPAL, XACML [2] and Hyperproperties [8]. These languages rely on the premise that the policies will be specified in a particular description language and then translated to models or implemented in a way that the agents understand. The approach taken in the CR is the opposite — the existing security policies are abstracted and compared for compatibility.

While the above-mentioned access controls could work to a degree in dynamic coalitions, there is still a great focus on who can access what, instead of managing the information flow directly. The Common Representation that we propose in this paper is intended to translate access controls from the domain of subjects and objects to information-flow domain and analysing the impact of such flows directly. The information-flow domain allows us to reason how information is exchanged between agents in a coalition and what information agents might be privy to. 


\section{Common Representation}

Common Representation (CR) provides a fresh approach to a policy-neutral method of defining access control constraints for a dynamic system by focusing on the flow of information within that system. In the context of dynamic coalitions, this represents the information exchange between agents and resources. The CR also allows coalition architects to reason about and analyse the effects of permitting new interactions between agents on confidentiality and what the impact departures of agents have on availability of information.

The robustness of CR stems from an observation that groups of agents joining a coalition already have an access control policy guarding the interaction between them. Thus, to provide secure communication efficiently, merely providing a bridge between various groups of agents would suffice, deferring all access control to run-time.

\subsection{Elements of a CR}

$\mathrm{CR}$ only concerns itself with read and write privileges as these are the only ones that cause information to be exchanged. If any other access control modes lead to data flows, e. g. execute privilege, those, too, can be refined down to read and write operations.

A common representation is a directed graph $C R=(I, F)$ where interfaces, $I$, represent vertices and flows, $F \subseteq I \times I$, represent edges.

An interface is an abstract representation of a communication port. There are two types of interfaces: explicit and implicit. An explicit interface is an interface that represents a tuple of a resource and an access mode, e. g. $\left(r_{1},\langle\mathrm{w}\rangle\right)$; this type of interface an be either active (send/write) or passive (receive/read). An implicit interface can only represent an agent and can be both active and passive. Implicit interfaces are an important utility in modelling information flows where information is exchanged without a dedicated (or perceived) resource, for example, in face-to-face communication. Any agent can have numerous implicit interfaces. The choice of what type of interface to use largely depends on the desired level of abstraction required by the security policy, although the decision has repercussions on the operations as well as the ability to analyse resulting information flows.

Flows in the $C R$ graph are used to represent permitted information exchange paths between interfaces and are expressed as pairs of interfaces. A read from $i_{a}$ to $i_{b}$ would be expressed as $\left(i_{a}, i_{b}\right)$ while a write from $i_{a}$ to $i_{b}$ would be expressed as $\left(i_{b}, i_{a}\right)$. Each flow is asymmetric and if a flow $\left(i_{a}, i_{b}\right)$ exists, there is no implication of a complementary flow $\left(i_{b}, i_{a}\right)$. The flows are always expressed left-to-right,

i. e. $\left(i_{\text {from }}, i_{t o}\right)$. Any flow expressed as $\left(i_{a}, i_{b}\right)$, could mean either $i_{a} \underset{\text { from }}{\stackrel{\text { read }}{\text { rom }}} i_{b}$ or $i_{b} \underset{\text { to }}{\stackrel{\text { write }}{\longrightarrow}} i_{a}$, which are equivalent. This equivalence and asymmetry means that a $\mathrm{CR}$ will require two flows to represent a bidirectional exchange of data: $\left(i_{a}, i_{b}\right),\left(i_{b}, i_{a}\right)$. Bi-directional flows are called complementary - two flows $f$ and $f^{-1}$ are complementary if and only if $f \equiv\left(i_{a}, i_{b}\right) \wedge f^{-1} \equiv\left(i_{b}, i_{a}\right)$.

Directed representation of flows has a notable side effect - "read" and "write" lose their semantic meaning when converted into information flows - the only way that the original nature of a particular flow would be preserved is if at least one of the interfaces is explicit; this highlights the importance of not relying on implicit flows.

Access, with respect to the access control policies, is an operation which leads to information being exchanged between objects and subjects. Thus by expressing the common representation as a directional graph of information flows we can reason about information access within the coalition.

The nature of any DC is that access can be granted or revoked on several occasions. This level of 
abstraction allows explicit interfaces to be destroyed and instantiated on-request (when an agent leaves the coalition for example) thus fulfilling the demand for dynamic changes in access controls to a resource. Additionally, each agent can have its own dedicated interface to each of the resources shared by other agents. Thus when an interface is destroyed because an agent leaves, other agents can still retain access to the same resources in the coalition.

Additionally, this representation allows any resource to have multiple interfaces, a concept which could be used to model polyinstantiation - an explicit interface would be a third order object in the mapping of $A \rightarrow R \rightarrow I$.

\subsection{Granting Access}

Securing the interaction between agents is based on the Clark-Wilson model [7] around a Trusted Computing Base (TCB) described by Lampson et al. [14]. The Clark-Wilson model refers to the notion of mediated access between an object and a subject: Subject $\longrightarrow$ Mediator $\longrightarrow$ Object and TCB brings about various requirements, like hardware and software support, for the mediator. The TCB refers to the mediator as a reference monitor, which in itself is an abstract representation. The reference monitor consists of numerous security kernels. Each security kernel is made of trusted and verified software and hardware. As these security kernels are verified and are trusted to perform within specification, only the security kernels are permitted to interact between agents. Therefore, removing the need to rely on correct behaviour of any agent.

Access, by definition, is a process where information flow occurs from $a$ to $b$. From this definition, granting access from one interface to another within the CR is simply the matter of verifying whether a particular flow is permitted.

In the absence of SMP and any other constraints, the default behaviour for a security kernel for granting access between to interfaces can be described by a simple function grant. For brevity, in this paper, we will assume that access will only be granted if the CR is authoritative over both interfaces, otherwise the result of the function is undefined. For a CR to be authoritative over an interface, the interface must be defined in the vertices of the CR graph.

$$
\begin{aligned}
& \text { grant }: I \times I \times C R \rightarrow \mathbb{B} \mid \text { undef } \\
& \operatorname{grant}\left(i_{1}, i_{2},\left(i_{s}, f_{s}\right)\right) \begin{cases}\left(i_{1}, i_{2}\right) \in f_{s} & \text { if }\left\{i_{1}, i_{2}\right\} \subseteq i_{s} \\
\text { undef } & \text { otherwise }\end{cases}
\end{aligned}
$$

\section{Representing Basic Policies}

The previous section gave an overview of the structure of common representation. This section will discuss how common access control policies like those described in Section 2 are mapped into common representation.

\subsection{Discretionary Access Controls}

Discretionary Access Controls are typically expressed as a Capability Lists or Access Control Lists (ACL), either of which is derived from an Access Control Matrix (ACM). An access control matrix is a table describing permitted interactions between objects $\left(o_{1}, o_{2}, o_{3}\right)$ and subjects $\left(s_{1}, s_{2}, s_{3}\right)$. An example ACM is shown in Table 1 . 


\begin{tabular}{c|ccc} 
& $o_{1}$ & $o_{2}$ & $o_{3}$ \\
\hline$s_{1}$ & $\mathrm{r}$ & $\mathrm{W}$ & $\mathrm{rw}$ \\
$s_{2}$ & - & $\mathrm{W}$ & $\mathrm{r}$ \\
$s_{3}$ & $\mathrm{rW}$ & - & $\mathrm{r}$
\end{tabular}

Table 1: Simple Access Control Matrix

The access control matrix is a form of discretionary access control where the permission mapping between subjects and objects remain fairly static and well-defined at any one time. The next two access controls fall into a non-discretionary access controls category where instead of having a clear definition of interactions, access is granted based on a set of predefined criteria.

The access control matrix shown in Table 1 can be described as an access control list:

$$
\begin{aligned}
\left\{o_{1}\right. & \mapsto\left\{\left(s_{1},\langle\mathrm{R}\rangle\right),\left(s_{3},\langle\mathrm{R}\rangle\right),\left(s_{3},\langle\mathrm{~W}\rangle\right)\right\}, \\
o_{2} & \mapsto\left\{\left(s_{1},\langle\mathrm{~W}\rangle\right),\left(s_{2},\langle\mathrm{~W}\rangle\right)\right\}, \\
o_{3} & \left.\mapsto\left\{\left(s_{1},\langle\mathrm{R}\rangle\right),\left(s_{1},\langle\mathrm{~W}\rangle\right),\left(s_{3},\langle\mathrm{R}\rangle\right)\right\}\right\}
\end{aligned}
$$

For any ACM that describes access controls between a set of subjects, $S$, and a set of objects, $O$, the DAC can be translated to a CR containing $O \cup S$ as the set of interfaces. The flows of the CR are based on the read-and write- operations permitted by the access controls. ACLs are expressed as mappings from objects to a set of subjects and access modes those subjects have to a particular object: ACL: $O \rightarrow \mathscr{P}(S \times M)$. Translating an ACL to CR once objects and subjects have been mapped to interfaces is only a matter of determining resulting

$$
\begin{gathered}
\{((s,\langle\mathrm{R}\rangle),(o,\langle\mathrm{~W}\rangle)) \mid o \in O,(s,\langle\mathrm{~W}\rangle) \in A C L(o)\} \\
\quad \cup \\
\{((o,\langle\mathrm{R}\rangle),(s,\langle\mathrm{~W}\rangle)) \mid o \in O,(s,\langle\mathrm{R}\rangle) \in A C L(o)\}
\end{gathered}
$$

Capability Lists can be translated into common representation in a similar manner.

\subsection{Lattice-Based Access Control}

A Lattice-Based Access Control policy typically consists of three components: subjects, objects and labels. Each subject and object is marked with a label, then the labels are organised into a partially ordered dominance hierarchy $\left(L, \leq_{L}\right)$, a special abstract function $\lambda: S \cup O \rightarrow L$ is then used to determine the label associated with each object or subject.

The most commonly used LBAC policy is the Bell-LaPadula security model [3]. The underlying principle of the model is that access is determined by the dominance relationship between the object and the subject and that the subjects and objects are interchangeable for the purpose of modelling. The model states that $A$ can write to $B$ and $B$ can read from $A$ if $\lambda(A) \leq_{L} \lambda(B)$. This model reflects the equivalence of flows in the common representation that was discussed earlier.

The derivation of the common representation for LBACs is similar that for DACs except that readand write- flows are treated differently. Let us assume that set $I$ is a union of $S$ and $O$. Total flows are derived as

$$
\left\{\left(i_{1}, i_{2}\right) \mid i_{1}, i_{2} \in I \cdot i_{1} \neq i_{2} \wedge \lambda\left(i_{1}\right) \leq_{L} \lambda\left(i_{2}\right)\right\}
$$




\subsection{Role-Based Access Controls}

Role-Based Access Controls differ from the access controls described above in that there the relationship between objects and subjects is not defined within the access control, instead, the subject acquires privileges based on the role assignment. The implication of this phenomenon is that while it remains possible to derive the flows of information within each role across different roles, the representation of information exchange between subjects is deferred until the subject is assigned a particular role. Additionally, there is a notion of role seniority, where a senior role absorbs permissions of junior roles associated with it.

There are two components that are involved in expressing an RBAC: role assignments and role hierarchies. The role assignments consist of a mapping from a role to the privileges that role grants: $R A: R \rightarrow \mathscr{P}(O \times M)$. The role hierarchy is represented with the role seniority relationship: $R H \subseteq R \times R$. Due to this seniority relationship, it is necessary to determine transitive closure of each role described by the hierarchy. This can be done using Warshall's algorithm [19] or a similar algorithm described by Osborn [15], which is more closely related to role hierarchies; let the transitive closure over $R H$ be $R H^{+}$. The mapping of senior roles, seniority: $R \rightarrow \mathscr{P}(R)$, to junior roles can then be obtained through:

$$
\text { seniority }=\left\{r_{j} \mid\left(r, r_{j}\right) \in R H^{+}\right\}
$$

we can then go on to determine what privileges a specific role gives using privileges: $R \rightarrow \mathscr{P}(O \times$ Mode $)$ :

$$
\text { privileges }=\bigcup\left\{R A\left(r_{j}\right) \mid r_{j} \in \operatorname{seniority}(r)\right\} \cup R A(r)
$$

and finally, we can derive the flows that arise in the RBAC based on roles and the role hierarchy:

$$
\text { flows }=\bigcup\{\{((o,\langle\mathrm{R}\rangle),(o,\langle\mathrm{~W}\rangle)) \mid(o,\langle\mathrm{R}\rangle),(o,\langle\mathrm{~W}\rangle) \in \operatorname{privileges}(r)\} \mid r \in R\}
$$

\section{Compositionality of CRs}

Having looked at how individual access control policies can be translated into the common representation, we can now look at how these common representations can be combined together to form an overall common representation of information flow for the whole of a dynamic coalition.

All operations on CRs assume that the names of the interfaces in all CRs, which are involved in a particular operation, are named consistently. That is, if $C R_{1}$ contained interface named $i_{a}$ and $C R_{2}$ contained interface named $i_{a}$, then both CRs refer to the same, identical interface.

There are two composites that can be formed from a collection of common representations - the Simple Composite and the Priority Composite. Both operations have equal precedence. Thus it is important to explicitly group expressions containing both of these composites.

\subsection{Simple Composite - Merge}

When two groups of agents join to form a coalition, their respective common representations need to be merged together to form a coalition-wide representation of information flows. The most likely scenario for a coalition formation is when the agents in separate groups are isolated from other groups in terms of access privileges. This merge is achieved by performing a simple composite.

The simple composite is the most straightforward way of combining numerous common representations. This composite favours permit-type flows and will effectively result in a less strict policy overall 
compared to any individual common representation.

$$
\begin{aligned}
& \text { merge: } C R \times C R \rightarrow C R \\
& \text { merge }\left(\left(i_{a}, f_{a}\right),\left(i_{b}, f_{b}\right)\right) \triangleq\left(i_{a} \cup i_{b}, f_{a} \cup f_{b}\right)
\end{aligned}
$$

This merge operation will result in a graph of order two or higher, if the two merging CRs do not have any interfaces in common, as a case with independent All simple composites have commutative properties when applied as a distributed operation over a set of common representations.

\subsection{Priority Composite - Append}

Numerous times during the evolution of a dynamic coalition, a group of unassociated agents will inevitably join an existing coalition. When a group of agents joins in, the flow restrictions defined by the established coalition must be respected, thus any group that is merging-in will only create flows to the additional agents. This type of merging is called a priority composite, with priority given to one common representation. The priority composite is a way of combining common representations which favours deny over permit. The priority composite will not allow new flows to be created for existing interfaces where there does not exist a flow already.

$$
\begin{aligned}
& \text { append: } C R \times C R \rightarrow C R \\
& \text { append }\left(\left(i_{a}, f_{a}\right),\left(i_{b}, f_{b}\right)\right) \triangleq\left(i_{a} \cup i_{b}, f_{a} \cup\left\{f \mid f \in f_{b} \cdot\left\{f, f^{-1}\right\} \cap f_{a}=\emptyset\right\}\right)
\end{aligned}
$$

The priority composite is a non-commutative operation and operates over an ordered set of common representations with a left-to-right order.

\section{Analysing CRs and Composite Properties}

In this section we will look at how CRs can be analysed and look at how we might determine conflicts.

We can use a CR to determine the degree of availability of information flow paths within a DC using simple graph theory. For example, in determining whether there is a potential for information to be exchanged between two agents, we can check whether there exists a walk between the relevant interface of said agents. This is an important aspect when determining whether a particular interface is critical to the operation of the DC. We can derive the availability graph $C R^{A}$ of $C R$ by replacing all complementary flows in $C R$ with an edge in $C R^{A}$. The availability graph is an undirected graph and serves the purpose of determining whether information can flow between the interfaces of the DC. If the number of components in $C R^{A}$ is equal to one then we say that the liveliness property of a DC holds.

We will now proceed to look at conflicts in CRs. A CR conflict is said to occur when one common representation contains a flow between any two interfaces, and the second one does not contain a flow between the corresponding interfaces.

\subsection{Identifying Conflicts}

The first step to analysing any composite is to be able to identify conflicts between different common representations.

$$
\begin{aligned}
& \text { conflicting: } C R \times C R \rightarrow \mathbb{B} \\
& \text { conflicting }\left(\left(i_{a}, f_{a}\right),\left(i_{b}, f_{b}\right)\right) \triangleq \exists i_{1}, i_{2} \in\left(i_{a} \cap i_{b}\right) \cdot i_{1} \neq i_{2} \wedge\left(i_{1}, i_{2}\right) \notin\left(f_{a} \cap f_{b}\right)
\end{aligned}
$$




\subsection{Analysing Conflicts}

We can further expand on conflict resolution by zooming in on specific properties of CRs. For example, we can identify all flows that are permitted in interfaces $a$ but denied in $b$ are determined by expression in (6.2). Note that, in this case, a conflict may only arise if both CRs include identical interfaces. For example, given two CRs

$$
C R_{1}=\left(\left\{i_{a}, i_{b}, i_{c}\right\},\left\{\left(i_{a}, i_{c}\right),\left(i_{b}, i_{c}\right)\right\}\right)
$$

and

$$
C R_{2}=\left(\left\{i_{a}, i_{c}, i_{d}\right\},\left\{\left(i_{a}, i_{d}\right),\left(i_{d}, i_{c}\right)\right\}\right)
$$

the only conflict that arises is $\left(i_{a}, i_{c}\right)$, as $i_{b}$ is not a vertex in $C R_{2}$ and $i_{d}$ is not a vertex in $C R_{1}$.

$$
\begin{aligned}
\text { conflicts: } & C R \times C R \rightarrow F \\
\operatorname{conflicts}( & \left.\left(i_{a}, f_{a}\right),\left(i_{b}, f_{b}\right)\right) \triangleq \\
& \left\{\left(i_{1}, i_{2}\right) \mid \forall i_{1}, i_{2} \in\left(i_{a} \cap i_{2}\right) \cdot i_{1} \neq i_{2} \wedge\left(i_{1}, i_{2}\right) \notin\left(f_{a} \cap f_{b}\right)\right\}
\end{aligned}
$$

Similarly, flows common to both $a$ and $b$ can be identified from the intersection of edges of CR graphs $a$ and $b$. Finally, the flows that are different to both $a$ and $b$ :

$$
\begin{aligned}
& \text { diffs: } C R \times C R \rightarrow F \\
& \operatorname{diffs}\left(\left({ }_{-}, f_{a}\right),\left({ }_{-}, f_{b}\right)\right) \triangleq\left(f_{a} \cup f_{b}\right)-\left(f_{a} \cap f_{b}\right)
\end{aligned}
$$

\subsection{Compositionality Policies}

With the aid of algebraic operations described above, coalition architects can define compositionality policies which can then be used during the lifetime of the coalition to automate the process of assimilating groups of agents into the coalition without having to suspend operations while policies are integrated. Such policy may state, for example, "Conflicting policies $\boldsymbol{A}$ and $\boldsymbol{B}$ may be combined if the conflicts are limited to complementary flows of $\boldsymbol{A}$, otherwise, the composite policy must not create extra permissions than those already defined by $\boldsymbol{A}$ ". This can be expressed as:

$$
\begin{aligned}
& \text { if } \forall f \in \operatorname{conflicts}(A, B) \cdot \exists f^{\prime} \in \text { flows }(A) \cdot f^{\prime}=f^{-1} \\
& \text { then } \operatorname{merge}(A, B) \\
& \text { else append }(A, B)
\end{aligned}
$$

where flows is an abstract function determining information flows within a graph.

Defining policies like these is one of the purposes for the Security Meta-Policy framework which takes the primitives of access controls and security policies and describes ways to constrain them and perform operations on policy-neutral representations in order to expedite the integration of new agents, impose coalition-wide constraints on policies and provide a mechanism for evaluating the effects of policy manipulation.

\section{Conclusion and Future Work}

In this paper we looked at a novel way of modelling security using a CR, how a CR is structured and discussed ways of representing various access control policies in the information-flow domain. We also 
have demonstrated how a CR can be used to address availability and confidentiality of the CIA security triad. Once the policies are expressed in CR, we looked at various operations for composition and analysis of policies.

The CR preserves the access constraints expressed in access control and security policies by design and compositional algebra, provides a way of manipulating not only different, but also conflicting policies. While this approach is sufficient for simple problems, dealing with more advanced concepts, like Separation of Duty or Brewer-Nash Models, requires the use of additional layer of parametrisation over the CR as well as the ability to define conditional evaluation operations. This layer is provided by the Security Meta-Policy Framework which is an ongoing work. The CR is put to use in the Security Meta Policy where the CR expression algebra is used for determining effects of changes in dynamic coalitions.

When the CR combined with a Security Meta-Policy, there exists a possibility to group interfaces by semantic meaning together to form blocks, modifying the definition of $C R$ to $C R:(B, F)$ where $B \subseteq \mathscr{P}(I)$ and $F \subseteq \mathscr{P}(B \times B)$. Each block can represent, for example, a set of interfaces that have unrestricted flow of information between each other, or a set of interfaces where flows are forbidden, or where the number of flows is limited between the interfaces from a block to any other block. This also allows us to model interactions that were previously not possible in other access control methods, like groups of people.

Additionally, given that CR is essentially a graph, it should be possible to define logic for optimisation of flows, thus reducing the number of unnecessary edges which in turn enforces the principle of least privileges. Another way of optimising the CR would be to group various complimentary interfaces together into individual blocks (enabled by the use of SMP). This approach would reduce the complexity of operations but would have a side effect of limiting the ability to precisely express details of interaction.

Coming back to the assumptions made in Section 2, we can now see how the atomicity of agents and granularity of tasks would affect the structure of the CR. However, this should not be a problem when translating existing security policies into $\mathrm{CR}$, as the desired levels of atomicity and task granularity should already be pre-defined in the source policies. When new tasks are created, or agents added, and a new CR is generated to include the desired changes, the new CR can be checked for conflicts against the existing CR using techniques described in Section 6. Results of such checks can be used to gauge whether there needs to be a further division of tasks or whether a particular flow is too permissive. This logic can also be included into the SMP to automate the decision making process and change configuration of the DC on-the-fly.

Finally, work on CRs could be linked with work on insider threat modelling described by Chinchani et al [6, 12]. These models, at the most fundamental level, rely on interaction graphs between different nodes of network to determine what node is exposed to what information. Such approach is similar to what common representation provides, thus by moving elements of insider threat modelling into the common representation, the risk of insider abuse could be analysed and mitigated.

Acknowledgements: We are grateful to Anirban Bhattacharyya, Jeremy Bryans, Peter Ryan and Alexander Romanovsky for many helpful discussions and valuable insights.

\section{References}

[1] J. P. A. Almeida, M. van Sinderen \& L. Nieuwenhuis (2001): Transparent Dynamic Reconfiguration for CORBA. In: DOA '01: Proceedings of the Third International Symposium on Distributed Objects and Applications, IEEE Computer Society, p. 197. 
[2] A. H. Anderson (2006): A comparison of two privacy policy languages: EPAL and XACML. In: SWS '06: Proceedings of the 3rd ACM workshop on Secure web services, ACM, pp. 53-60.

[3] D. Elliot Bell \& Leopard J. LaPadula (1973): Secure Computer Systems: Mathematical Foundations. Technical Report TR-2547, MITRE Corporation.

[4] J. W. Bryans, J. S. Fitzgerald, C. B. Jones \& I. Mozolevsky (2006): Formal Modelling of Dynamic Coalitions, with an Application in Chemical Engineering. In: T. Margaria, A. Philippou \& B. Steffen, editors: IEEEISoLA 2006: Second International Symposium on Leveraging Applications of Formal Methods, Verification and Validation, pp. 90-97.

[5] J. W. Bryans, J. S. Fitzgerald \& P. Periorellis (2007): A Formal Approach to Dependable Evolution of Access Control Policies in Dynamic Collaborations. In: Proc. 37th Annual IEEE/IFIP Intl. Conf. on Dependable Systems and Networks, pp. 352-353.

[6] R. Chinchani, A. Iyer, H. Ngo \& S. Upadhyaya (2005): Towards a Theory of Insider Threat Assessment. In: Proceedings of the International Conference on Dependable Systems and Networks, pp. 108-117.

[7] D. D. Clark \& D. R. Wilson (1987): A Comparison of Commercial and Military Computer Security Policies. In: IEEE Symposium on Security and Privacy, IEEE Computer Society, p. 184.

[8] Michael R. Clarkson \& Fred B. Schneider (2008): Hyperproperties. In: CSF '08: Proceedings of the 2008 21st IEEE Computer Security Foundations Symposium, IEEE Computer Society, Washington, DC, USA, pp. $51-65$.

[9] N. Damianou, N. Dulay, E. Lupu \& M. Sloman (2001): The Ponder Policy Specification Language. In: Lecture Notes in Computer Science, Springer-Verlag, pp. 18-38.

[10] Eric Freudenthal, Edward Keenan \& Vijay Karamcheti (2002): dRBAC: Distributed role-based access control for dynamic coalition environments. In: In Proceedings of the Twenty-second IEEE International Conference on Distributed Computing Systems (ICDCS), pp. 411-420.

[11] F. Freudenthal, E. Keenan, T. Pesin, L. Port \& V. Karamcheti (2001): DisCo: A Distributed Infrastructure for Securely Deploying Decomposable Services in Partially Trusted Environments. Technical Report 2001-820, Computer Science, New York University.

[12] D. Ha, S. Upadhyaya, H. Ngo, S. Pramanik, R. Chinchani \& S. Mathew (2007): Insider Threat Analysis using Information-Centric Modelling. In: Advances in Digital Forensics III, Springer, pp. 55-73.

[13] J. Kramer \& J. Magee (1990): The Evolving Philosophers Problem: Dynamic Change Management. IEEE Transactions on Software Engineering 16(11), pp. 1293-1306.

[14] B. Lampson, M. Abadi, M. Burrows \& E. Wobber (1992): Authentication in distributed systems: Theory and practice. ACM Transactions on Computer Systems 10, pp. 265-310.

[15] S. L. Osborn (2002): Information flow analysis of an RBAC system. In: 7th ACM Symposium on Access Control Models and Technologies, ACM Press, pp. 163-168.

[16] R. S. Sandhu, E. J. Coyne, H. L. Feinstein \& C. E. Youman (1996): Role-Based Access Control Models. Computer 29(2), pp. 38-47.

[17] Ravi S. Sandhu (1993): Lattice-Based Access Control Models. Computer 26(11), pp. 9-19.

[18] D. B. Stewart, R. A. Volpe \& P. K. Khosla (1997): Design of Dynamically Reconfigurable Real-Time Software Using Port-Based Objects. IEEE Trans. Softw. Eng. 23(12), pp. 759-776.

[19] S. Warshall (1962): A Theorem on Boolean Matrices. Journal of the ACM 9(1), pp. 11-12. 